\title{
Amplifier design for EMG recording from stimulation electrodes during functional electrical stimulation leg cycling ergometry
}

\author{
Raafat Shalaby ${ }^{1}$, Thomas Schauer ${ }^{1, *}$, Wolfgang \\ Liedecke $^{2}$ and Jörg Raisch ${ }^{1,3}$ \\ ${ }^{1}$ Control Systems Group, Technische Universität Berlin, \\ Berlin, Germany \\ ${ }^{2}$ HASOMED GmbH, Magdeburg, Germany \\ ${ }^{3}$ Systems and Control Theory Group, Max Planck Institute \\ for Dynamics of Complex Technical Systems, \\ Magdeburg, Germany
}

\begin{abstract}
Functional electrical stimulation leg cycle ergometry (FESLCE), which is often used as exercise for people with spinal cord injury (SCI), has recently been applied in the motor rehabilitation of stroke patients. Recently completed studies show controversial results, but with a tendency to positive training effects. Current technology is identical to that used in FES-LCE for SCI, whereas the pathology of stroke differs strongly. Most stroke patients with hemiparesis are able to drive an ergometer independently. Depending on the degree of spasticity, the paretic leg will partially support or hinder movements. Electrical stimulation increases muscle force and endurance and both are prerequisites for restoring gait. However, the effect of FES-LCE on improving impaired motor coordination is unclear. To measure motor coordination during FES-LCE, an EMG-amplifier design has been investigated which suppresses stimulation artifacts and allows detection of volitional or reflex induced muscle activity. Direct measurement of EMG from stimulation electrodes between stimulation pulses is an important asset of this amplifier. Photo-MOS switches in front of the preamplifier are utilized to achieve this. The technology presented here can be used to monitor the effects of FES-LCE to adapt the stimulation strategy or to realize EMG-biofeedback training.
\end{abstract}

Keywords: artifact suppression; cycling; electromyography; functional electrical stimulation; stimulation electrodes; stroke rehabilitation.

\footnotetext{
*Corresponding author: Thomas Schauer, Control Systems Group, Technische Universität Berlin, Einsteinufer 17, 10587 Berlin, Germany

E-mail: schauer@control.tu-berlin.de
}

\author{
Introduction \\ Functional electrical stimulation leg cycling \\ ergometry
}

Stroke is a major cause for disability and death in Western countries. In the European Union approximately one million first ischemic strokes occur each year [12]. The combination of an aging population, declining stroke case-fatality rates, and limited success in reducing the incidence of stroke has resulted in an increase in the prevalence of stroke survivors. Recovery of gait is one of the main goals of motor rehabilitation after stroke. Within this context, cycling on an ergometer represents an often used training modality to establish prerequisites for successful gait training. Such cycling exercises can also be safely performed from a wheelchair before gait training is possible. Modern ergometer systems possess electrical drives to support or hinder the cycling movement depending on the abilities of the patient. In one study [30], a cycling wheelchair is described which can be driven by pedaling with the legs. Using this wheelchair practical locomotion for the hemiplegic patients without walking ability is possible at least when moving on a straight line.

The authors believe that cycling exercises can prevent contractures of the leg joints and delay or prevent the onset of spasticity. Active cycling exercises also lead to an increase in endurance and muscle strength of both legs. These improvements lead to an increase of walking speed as reported in several studies [4, 17, 18, 21]. However, locomotor coordination does not improve post-intervention, as shown by Kautz et al. [18], for most of the patients. Only a very limited number of studies describe specialized cycling exercises for retraining of motor coordination.

Brown and DeBacher [3] proposed an EMG biofeedback cycling exercise to train muscle coordination in patients with spastic hemiparesis. The aim of the training is to increase the activity of the flexors in the paretic leg while decreasing spastic reactions of the leg extensors during flexion phases. The resistance of the ergometer is increased until the patient can control his/her paretic muscles in such a way that the $180^{\circ}$ phase shifted reciprocal muscle activity of the healthy leg can be reproduced by the paretic leg. A case study illustrated this approach.

Commercially available ergometers with motor assistance sometimes offer a symmetry training to balance the effort of paretic and healthy leg. A symmetry index is calculated from the motor torque and displayed to the patient who can adjust 
his/her cycling accordingly. However, because only the motor torque is used to determine the symmetry index and not individual force sensors at each pedal, the accuracy of such a symmetry index is questionable. Pulling and pushing with the healthy leg can equally lead the patient to believe the symmetry between both legs is perfect, whereas the paretic leg might not be active at all.

The application of functional electrical stimulation (FES) via surface electrodes to the lower limbs, with FES synchronized to the cycling movement, can enhance the rehabilitation progress of the patients. FES directly activates the paretic muscles. This prevents muscle atrophy and helps to build up muscle mass and strength. The possible gain in induced force due to FES in the paretic leg is generally limited because residual sensation limits the stimulation intensity. Therefore, a complete compensation for motor deficits by FES might not be possible in all stroke patients.

FES induced afferent-efferent stimulation together with cutaneous and proprioceptive inputs could be important in "reminding', subjects how to perform the movement properly. Indeed, this complete afference of the task could enhance the synaptic controls and modify the excitability of specific motor neurons, thus facilitating the reorganization of the motor schemes and accelerating the process of functional recovery. Such effects of FES have already been described in the literature concerning the stimulation of the upper extremity and FES for drop foot treatment in stroke survivors [33].

Several clinical studies [8, 9, 14, 35] evaluating the effects of FES leg cycle ergometry (FES-LCE) in gait rehabilitation of stroke patients have already been performed. Some of the studies report statistically significant positive effects on muscle force and statistically non-significant positive effects on some mobility measurements compared with standard rehabilitation [9] or cycling exercise without FES [8]. Janssen et al. [14] could not observe improvements in the training effects by adding FES to cycling exercises in stroke patients. The stimulation and training protocols differ much for all reported studies. This reveals that no standard protocols for FES cycling currently exist. Eigler [8] and Ferrante et al. [9] used motorized ergometers and stimulated both legs (also the non-paretic), whereas Janssen et al. [14] used an isotonic cycle ergometer (Ergys2) with flywheel stimulating only the paretic leg. Patients were allowed to cycle voluntarily on the resistance controlled ergometers (isotonic mode) in studies by Eigler [8] and Janssen et al. [14], whereas Ferrante et al. [9] instructed the patients to be passive during the exercise. In the latter case, a movement of the legs was solely induced by the motor and FES. Stimulated muscles in all studies have been the quadriceps, hamstring and M. gluteus maximus. In $[8,9]$, M. tibialis anterior was also electrically stimulated. In summary, recent studies show that FES assisted cycling exercise can have positive effects in stroke patients.

While muscle strength and endurance can be increased by such therapy forms, the effects on motor relearning and muscle coordination remain unclear. By extrapolating results from training programs with intensive ergometer exercises without stimulation, no positive effects could be expected.
Changes in muscle coordination due to the FES cycling rehabilitation program have not been evaluated using electromyograms (EMGs), neither during cycling nor during gait. Incorrect muscle activation patterns caused by spastic hemiplegia might be manifested by repeating such impaired movement patterns. In the study of Ferrante et al. [9], the symmetry in the task was the aim of the therapy. Therefore, both legs were stimulated and no voluntary muscle activity was requested from the patients. Using this approach, no incorrect movement patterns are elicited, which can yield good motor relearning and muscle coordination, as indicated by the presented results of a sit-to-stand test in [9].

Existing clinical FES cycling systems do not observe the immediate effect of the intervention on movement symmetry, muscle coordination, and spasticity. There is also no method to check if muscle fatigue is present or not. Ambrosini et al. [1] investigated the use of individual force sensors on both legs to exactly assess symmetry in the driving torques produced by FES. Based on this information, a feedback control strategy is proposed to individually adjust stimulation intensity applied to both legs with the aim of maintaining symmetry in torque generation.

Chen et al. [5] present an EMG driven stimulation system for FES cycling. The stimulation intensity is controlled proportionally to the detected "volitional" EMG activity. The latter is measured from separate EMG electrodes located on the muscle belly along its longitudinal axis at mid-distance between the stimulation electrodes. This approach, however, considerably increases the number of electrodes to attach. It should be noted that the proposed method simply distinguishes between correct and incorrect muscle activation by comparing the activities of both legs to avoid activation of electrical stimulation due to incorrect muscle activation.

This study describes developments towards a new EMG amplifier system which enables EMG recordings directly from the stimulation electrodes also during stimulation. The gained information on motor coordination can be used to adjust parameters in FES-LCE or to provide a visual biofeedback to the patient. In the latter case, electrical stimulation is indicating the "correct" intervals during which the patient should control his/her muscles.

\section{EMG recording combined with FES}

In clinical practice, EMG-triggered stimulation is often used to improve brain relearning by reinstating proprioceptive feedback time-locked to each attempted movement $[2,22]$. The movement intention is detected from EMG recordings using the stimulation electrodes while stimulation is not active. As soon as a patient exceeds a predefined level of EMG activity the electrodes are switched from the EMGamplifier input to the stimulator output. Afterwards, a predefined stimulation pattern (pulse train) will be applied for a fixed time period to the patient. During this time interval there is no EMG recording possible. Several commercial devices for EMG-triggered stimulation are on the market.

The detection of non FES-induced EMG activity (related to volitional movements or reflexes) during electrical stim- 
ulation (between the stimulation pulses) is problematic as large measurement artifacts occur [23]. The electrical stimulation pulses cause a stimulation artifact which can drive the EMG amplifier into saturation or even damage the amplifier. Parameters of electrical stimulation pulses used in FES cycling with stroke patients are as follows: current controlled bipolar pulses, pulse widths up to $500 \mu \mathrm{s}$ (one phase), stimulation frequency of $20 \mathrm{~Hz}$, and current amplitudes up to $50 \mathrm{~mA}$. To deal with the problem of stimulation artifacts, the EMG amplifier can either be disconnected from measurement electrodes (muted) when applying stimulation pulses $[15,16,19,25,26,29]$ or must provide a very fast recovery from input overloads [36]. After delivery of an electrical stimulation pulse, an asymmetrical charge can remain under the measurement electrodes. The related discharging process causes another measurement artifact. This artifact will be larger if EMG is directly measured from stimulation electrodes also due to their larger size. For this reason, almost all existing systems are using separate electrodes for stimulation and EMG measurement.

Another problem in detecting volitional muscle activity and/or reflex activity represents the so-called M-wave resulting from a synchronous contraction of several motor units induced by the electrical stimulation pulses. The amplitude of this EMG signal part is generally in the range of some $\mathrm{mV}$, whereas the intensity of the volitional/reflex EMG components is usually in the range of $\mu \mathrm{V}$. The M-wave starts a couple of milliseconds after the stimulus and has its main activity within $20 \mathrm{~ms}$ after the stimulus. This artifact superposes the volitional/reflex related EMG which is of interest.

Approximately 20-30 ms after a stimulation pulse, usually only lower frequent stimulation artifacts remain (tails of discharging curve and M-wave) in the measured signal. These artifacts can be eliminated by applying a high-pass filter with a cut-off frequency reported in the range of 100-330 Hz $[26,29,39]$. It should be noted that most of the spectral energy of the EMG is located between 30 and $300 \mathrm{~Hz}$ with a peak around $120 \mathrm{~Hz}$ [6]. Thus, only higher frequency parts of the volitional/reflex EMG are taken into account by applying such a high-pass filter. In the resulting signal, the period with filter transients and remaining stimulation artifacts is usually blanked (signal set to zero) before carrying out the standard EMG processing (rectification followed by a lowpass filter). The blanking period is usually from the onset of the stimulation pulse to approximately $20-30 \mathrm{~ms}$ after delivery of the stimulation pulse. Having a stimulation period of $50 \mathrm{~ms}$, for example, approximately $20-30 \mathrm{~ms}$ of volitional/ reflex related EMG signal can be obtained.

If no other artifacts hide M-wave and non-FES induced EMG then the M-wave can be estimated from EMG recordings of past stimulation periods under the assumption that M-wave and volitional/reflex related EMG are not correlated $[10,20,31,42]$. Hence, the latter can be determined by subtracting the estimated M-wave from the amplifier output. This represents an often used alternative to the high-pass filter approach outlined before. However, this requires complex digital signal processing. A simple but not very precise solution for direct estimation and extraction of the M-wave within one step is to use a comb filter which subtracts the identical sample of the previous stimulation period from the actual measurement during a stimulation period [10].

Several clinical studies have been published investigating the detection and use of voluntary induced EMG activity during FES [5, 11, 13, 15, 37-40]. The aim of all studies was to amplify a weak movement intention of the patient by an electrical stimulation proportional to the measured volitional EMG. In most cases, single channel EMG detection and stimulation systems were employed using five electrodes: two separate pairs of electrodes, one for stimulation and one for EMG detection, plus a single reference electrode. Extending this approach to multiple target muscles is not practical because of the large number of electrodes involved. Also, attaching four surface electrodes for EMG and FES over small target muscles might be challenging.

Only a very limited number of articles describing EMGrecording systems from stimulation electrodes using switching circuits has been reported $[15,16,26]$. Kamono et al. $[15,16]$ developed a device for gait assistance that applies electrical stimulation via the EMG electrodes. The stimulation pulses were repeated at a rate of $15 \mathrm{~Hz}$, and a microcontroller was used to switch electrodes between stimulation circuit and EMG amplifier. The authors did not provide detailed information about the timing sequence and/or measurement artifacts. However, from the low-stimulation frequency it can be speculated that stimulation artifacts last so long that the standard stimulation frequency of $20 \mathrm{~Hz}$ could not be used. The large interval between stimulation pulses of $66.7 \mathrm{~ms}$ was probably selected to obtain sufficiently long artifact-free EMG recording intervals. This solution is not acceptable in FES cycling because stimulation frequencies of $20 \mathrm{~Hz}$ and above are used.

Muraoka [26] developed a similar device that detects a volitional EMG interval of up to $25 \mathrm{~ms}$ from a stimulation period of $50 \mathrm{~ms}$, but for a rather narrow frequency band $(330-460 \mathrm{~Hz})$. During stimulation the stimulator is directly connected to the preamplifier terminals with the amplifier gain being set to zero. During measurement, the voltage controlled stimulator is disconnected from the stimulation electrodes. One possible reason for this action could be the low-output resistance of the stimulator stage which could attenuate the EMG measurement.

Both devices have only been applied for stimulation of muscles which only require moderate stimulation intensity (dorsiflexor in $[15,16]$ and upper limb muscles in [11, 13, 26]). It is therefore questionable if these EMG amplifiers work adequately for higher stimulation intensities applied during FES cycling in stroke patients. The amplifier presented in this paper is an extension of the amplifier presented previously [32]. Switching circuit and filtering stages have been adapted to allow EMG recordings within the context of FES cycling. The amplifier was extended to four channels and combined with the FES cycling system described in [28]. In contrast to the work in $[16,26]$, a current controlled stimulator with high-output resistance and a different switching circuit were used. 


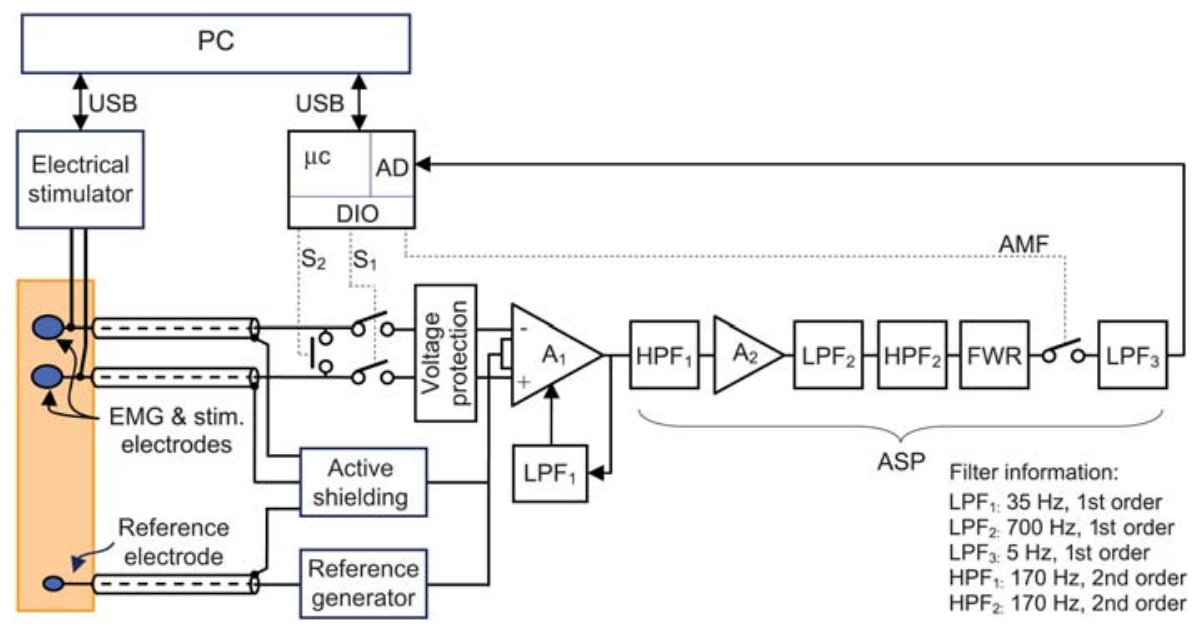

Figure 1 Stimulation and EMG measurement system with shared electrodes (only one channel is illustrated).

\section{Material and methods}

\section{EMG amplifier}

A four-channel EMG amplifier has been designed to measure EMG through stimulation electrodes during FES cycling. The block diagram depicted in Figure 1 shows the different amplification, filtering, and muting stages for one channel.

\section{EMG preamplifier}

The first amplification stage $\left(\mathrm{A}_{1}\right)$ utilizes the instrumentation amplifier INA129 (Texas Instruments, Dallas, TX, USA) as a preamplifier. For the whole EMG amplifier, a common mode rejection ratio (CMRR) of at least $80 \mathrm{~dB}$ has been experimentally determined for common mode disturbances up to $700 \mathrm{~Hz}$ while the measured input resistance is 130 $\mathrm{M} \Omega$. The preamplifier also possesses a very low offset voltage $(50 \mu \mathrm{V})$. INA129 is furthermore supplemented with integrated overvoltage protectors at input terminals. This protection can withstand only up to $\pm 40 \mathrm{~V}$ without damage. Protection against stimulation pulses of up to $160 \mathrm{~V}$ is therefore introduced by the later described switching circuit. Furthermore, a resistor diode circuit is clamping the input signal to the $\pm 5 \mathrm{~V}$ supply voltages provided by $8 \mathrm{AA}$ batteries. This feature is very useful as it protects the preamplifier in the case of an unwanted direct connection of stimulator outputs and amplifier inputs. Such an event could be caused by a failure in the switching circuit. The resistor diode circuit consists of diodes (BAV199, NXP Semiconductors, Eindhoven, The Netherlands) with very low leakage current (typically $9 \mathrm{pA}$, max. $5 \mathrm{nA}$ ) and thin-film precision chip resistors (Tyco Electronics, Berwyn, PA, USA) with $0.1 \%$ tolerance. The amplifier has at least $100 \mathrm{~dB}$ power supply rejection ration (PSRR) for frequencies up to $60 \mathrm{~Hz}$. No variations in the supply voltage above this frequency are expected as batteries operate the amplifier.

The preamplifier has first order high-pass characteristics (cut-off frequency of $35 \mathrm{~Hz}$ ) in order to suppress movement and stimulation artifacts from the input signal. As shown in
Figure 1, this AC-coupling has been realized by feeding the output of the instrumentation amplifier through a first order $35 \mathrm{~Hz}$ low-pass filter $\mathrm{LPF}_{1}$ back to its reference terminal as proposed in the INA129 data sheet. The preamplifier gain was designed to be $10 \mathrm{~dB}$. The stability of the AC-coupled instrumentation amplifier feedback loop (formed by $\mathrm{A}_{1}$ and $\mathrm{LPF}_{1}$ in Figure 1) was analyzed using standard linear system theory. The blanking procedure was not included in the stability analysis.

A switching circuit described in the next section protects $A_{1}$ from any damage caused by the stimulation pulses under normal operation.

\section{Switching network}

For each amplifier channel two sets $S_{1}$ and $S_{2}$ of photo-MOS switches AQV253 (Matsushita Electric Works Ltd., Osaka, Japan) have been implemented at the amplifier front-end (Figure 1). The photo-MOS switch AQV253 was used due to its capability to control an extremely high load voltage, up to $250 \mathrm{~V}$, using a low forward LED current (typically $0.9 \mathrm{~mA}$ ) and its very low on-resistance (typically 5.5 Ohm). This low on-resistance ensures fast elimination of the charge under the electrodes via S2. Furthermore, this switch type possesses a high-speed switching behavior (worst case measured switching times: $0.95 \mathrm{~ms}$ turn-on and $0.1 \mathrm{~ms}$ turn-off time). The first set $S_{1}$ protects the input circuit of the EMG preamplifier from any damage by electrical stimulation pulses and reduces the transient artifact by completely isolating the amplifier input from both the current controlled stimulator as well as the target muscle during delivery of stimulation pulses. The used stimulator possesses two current sources which are multiplexed to four stimulation channels each. For the application considered here, each leg is connected to one stimulation module (current source) such that stimulation pulses to both legs are delivered in parallel. Note that in practice not all muscles are active during cycling at the same time. There is a time gap of $1.5 \mathrm{~ms}$ between stimulation pulses sent from one stimulation module to the dif- 


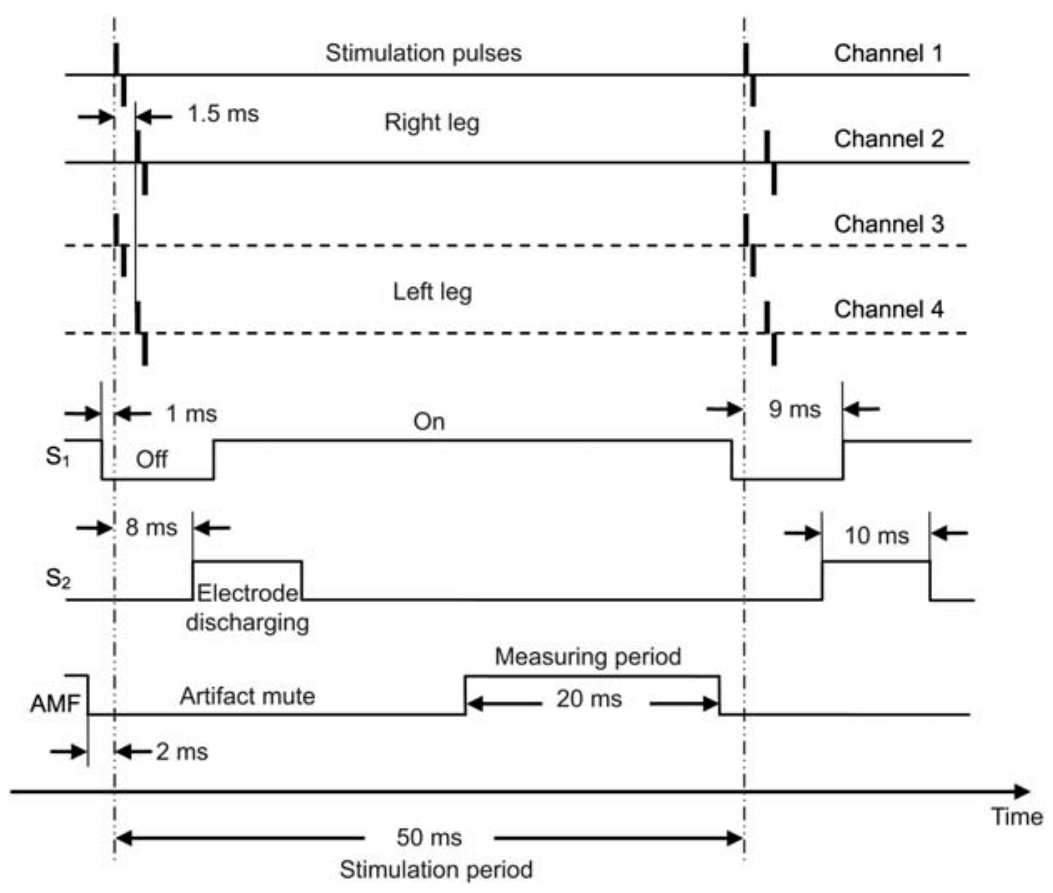

Figure 2 Timing chart for stimulation and amplifier photo-MOS switches.

ferent channels of the corresponding leg. The timing of the switches has been tuned under the assumption that only two muscles are stimulated on each leg during cycling. Figure 2 shows the timing of stimulation and switching for a stimulation period of $50 \mathrm{~ms}$. Under this assumption, the two switches $S_{1}$ are opened for $10 \mathrm{~ms}$, starting $1 \mathrm{~ms}$ before the delivery of stimulation pulses. When all stimulation pulses have been delivered, the set $S_{2}$ enables a fast recovery by introducing a very low-resistance current path causing fast electrode-skin discharging. This minimizes the length of the device blinding period due to saturation. All switches are controlled by external control signals generated on a microcontroller. Figure 2 also shows the operation of the artifact mute filter (AMF) described in the next section.

\section{Signal processing}

As shown in Figure 1, the output signal from the preamplifier $\mathrm{A}_{1}$ passes through a chain of analog signal processing (ASP) stages, containing filtering, scaling, and muting components. These ASP stages were implemented using the dual operational amplifier OP200 (Analog Devices, Norwood, MA, USA). Advantages of this amplifier are its low noise signal and high stability when driving a large capacitive load. The preamplifier is followed by a Chebychev configuration second order active high-pass filter $\left(\mathrm{HPF}_{1}\right)$ with unity gain and $170 \mathrm{~Hz}$ cut-off frequency. A Chebychev configuration has been selected to achieve a fast roll-off in the frequency response. The high-pass filtered signal is then rescaled by a gain of $61 \mathrm{~dB}$ through the amplifier stage $\mathrm{A}_{2}$ and low-pass filtered (first order filter $\mathrm{LPF}_{2}$ with cut-off frequency of $700 \mathrm{~Hz}$ ). High frequency noise is suppressed by this lowpass filter. After that filter, the signal is again high-pass fil- tered through $\mathrm{HPF}_{2}$ having the same characteristics as $\mathrm{HPF}_{1}$. The purpose of the second high-pass filter is to assure elimination of the DC part of the signal before analog full-wave rectification. Thus, the EMG amplifier has an entire gain of approximately $71 \mathrm{~dB}$, before rectification, and total bandpass characteristics of $170-700 \mathrm{~Hz}$ (sixth order). Additional ASP is performed via a precision full-wave rectifier followed by another photo-MOS switch AQV257 acting as an AMF. Figure 2 shows the timing signal of the AMF which introduces a muting period of $30 \mathrm{~ms}$. This period starts $2 \mathrm{~ms}$ before the delivery of stimulation pulses. Finally, the average value of the detected volitional/reflex muscle activity is obtained by using a $5-\mathrm{Hz}$ first order active low-pass filter $\left(\mathrm{LPF}_{3}\right)$.

The EMG amplifier output is sampled at 20 samples per second by a micro-controller ATmega328 (Atmel Corporation, San Jose, CA, USA) with a 10-bit A/D converter. Within the last filter stage $\left(\mathrm{LPF}_{3}\right)$, signal amplification by the factor 3.5 is performed to fully exploit the input range $(0-5 \mathrm{~V})$ of the A/D converter. The noise variance after the $\mathrm{A} / \mathrm{D}$ converter is $2.7 \mathrm{LSB}$. The sampled digital signal is transmitted via an optically isolated USB link to a PC as shown in Figure 1. Synchronization of the stimulation and EMG measurement (timing of switches and sampling) is in the responsibility of a PC program (running under real-time Linux) which periodically triggers stimulator and microcontroller via commands sent over optically isolated USB interfaces at the stimulation frequency of $20 \mathrm{~Hz}$.

\section{Active shielding and reference generator}

Active shielding is commonly used to reduce the effects of external noise and electrostatic interferences as well as 


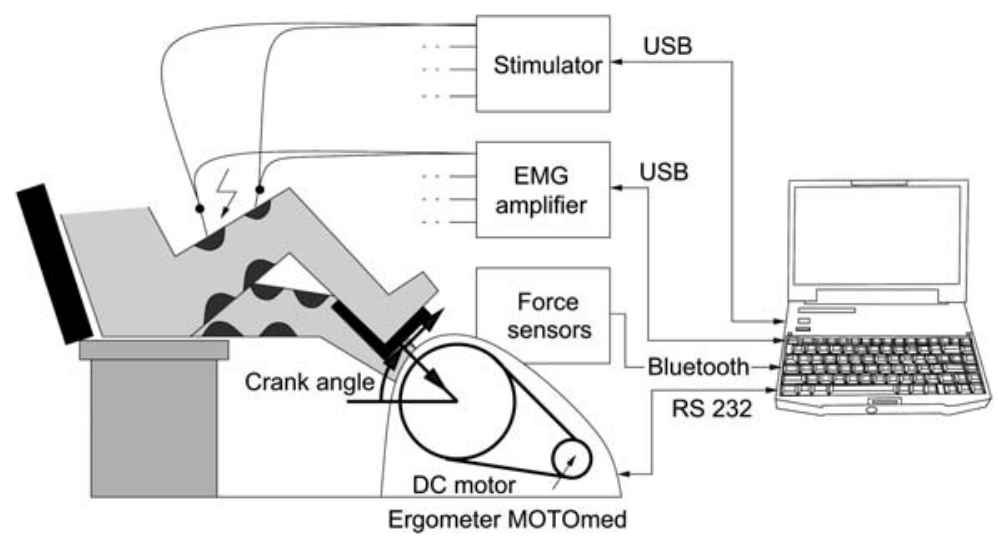

Figure 3 FES cycling system with integrated EMG measurement from stimulation electrodes.

effects of parasitic capacitances of the shielded cables. In the case of active shielding, the outer conductor (shielding) is simply driven at the average potential of the input terminals by using a buffer amplifier. Using active shielding, external interferences are suppressed through the low output impedance of the unity gain amplifier. Parasitic capacitances of the shielded cables for differential mode signals are not problematic as the measurement of EMG signals has restricted frequency content [24]. Each EMG channel has its own active shielding.

A single reference signal generator (driven-right leg system [41]) is used for all EMG channels to reduce common mode disturbances at the measurement electrodes up to several volts of the power supply of the amplifier. Input to this reference driver is the average of the input signals of the first channel.

For EMG measurement, carbon-coated shielded cables (Mind Media BV, Roermond-Herten, The Netherlands) have been used. Special adapters have been prepared to connect stimulation and EMG measurement cables at the electrode side.

\section{FES cycling system}

The EMG amplifier has been integrated into a computercontrolled FES cycling ergometer as shown in Figure 3. The basis of the system is a commercially-available cycling ergometer with functional electrical stimulation (RehaMove, HASOMED GmbH, Magdeburg, Germany), which consists of a motor-assisted ergometer (MOTOmed Viva2, RECKTechnik GmbH \& Co. KG, Betzenweiler, Germany) and an eight-channel neuromuscular electrical stimulator (RehaStim, HASOMED GmbH). Stimulated muscles are the hamstrings and quadriceps on both legs. Large reusable self-adhesive stimulation electrodes (type: RehaTrode, HASOMED $\mathrm{GmbH}$ ) of size $7.5 \times 13 \mathrm{~cm}$ are used to activate these muscles. The electrodes for the quadriceps were mainly placed to cover M. rectus femoris and M. vastus lateralis, whereas the hamstring electrodes are mainly located over the M. biceps femoris and M. semimembranosus. The ergometer can be parameterized externally via a serial link and provides periodic readings of cadence, crank angle, and motor torque. Possible modes of exercise are isokinetic (constant cadence) and isotonic (constant resistance) cycling. The stimulator can be controlled from a PC via an optically isolated USB interface using the ScienceMode protocol (http://sciencestim. sf.net).

The ergometer is equipped with two force sensors [34] (PowerTec, o-tec GmbH, Bensheim, Germany) to obtain measurements of radial and tangential forces acting on both crank arms in real time. Two OEM Bluetooth modules (Bluesense AD, Corscience GmbH \& Co. KG, Erlangen, Germany) are used for wireless data transmission of the force measurements. After each mounting of the forces sensors a calibration is required due to misalignment of the sensor axes and the coordinate system assigned to the crank arm. The calibration is performed by mounting cylindrical weights with known mass at the pedal threads and measuring the resulting forces for different constant cadences. Based on the expected force components for this load, the calibration is performed using the least squares method to calculate the scaling and bias as well as the rotation of the sensor coordinate system with regard to the crank arm for each sensor.

In the case of a constant cadence, controlled by the electrical motor, one can easily calculate the "active" forces at the crank arms produced by the leg muscles from the calibrated force recordings. This is achieved by subtracting the "passive" forces, i.e., mainly gravitational forces due to the mass of the legs, from measured radial and tangential forces. These forces which are dependent on the crank position must be determined initially during a phase of passive cycling where the legs are solely moved by the motor. Once determined the force profiles are stored in a look-up table. A symmetry index for active force generation of both legs can be calculated using the force sensors. To maintain symmetry in the FES induced forces of both legs, a feedback control method is presented by Ambrosini et al. [1]. The proposed method requires that the patient is passive during the entire FES cycling session and does not produce any non-FES related force. This basically means that patients with a marked spasticity and/or increased muscle tone as well as 
Table 1 Stimulation pattern defined by angular ranges of the crank for a cadence of $30 \mathrm{rpm}$.

\begin{tabular}{|c|c|c|c|c|}
\hline \multirow[t]{2}{*}{ Muscle } & \multicolumn{2}{|l|}{ Left leg } & \multicolumn{2}{|l|}{ Right leg } \\
\hline & Start angle $\left({ }^{\circ}\right)$ & Stop angle $\left({ }^{\circ}\right)$ & Start angle $\left({ }^{\circ}\right)$ & Stop angle $\left({ }^{\circ}\right)$ \\
\hline Quadriceps & 145 & 305 & 325 & 125 \\
\hline Hamstring & 315 & 105 & 135 & 285 \\
\hline
\end{tabular}

patients unable to relax are excluded from FES cycling using automatic symmetry control.

\section{Pattern generator}

The stimulation of the individual muscle groups is switched on and off depending on crank angle and cadence. Figure 3 shows the definition of the crank angle. A "static" stimulation pattern is defined which describes angular ranges in which a certain muscle will produce a positive tangential force at the crank arm. During cycling the muscles have to be activated earlier than defined in the static pattern as a time delay between stimulation and force generation of approximately $200 \mathrm{~ms}$ must be taken into account. The static stimulation pattern is therefore shifted proportionally with regard to the cadence to obtain a "dynamic" stimulation pattern. Within the activation ranges, an arbitrary stimulation profile, with the crank angle as argument, can be assigned. Currently, profiles are trapezoidal with ramps over $50^{\circ} \mathrm{crank}$ angle. The stimulation conditions are checked at a time interval of $50 \mathrm{~ms}$ corresponding to the stimulation frequency. The entire stimulation control is realized on a PC running realtime Linux (RTAI extension, http://www.rtai.org) under openFrameworks (http://www.openframeworks.cc) which is an open source $\mathrm{C}++$ toolkit for creative coding with OpenGL for graphics. This allows an almost effortless implementation of visual biofeedback strategies in FES cycling based on EMG.

\section{Charge control}

The stimulation intensity is determined by the frequency of stimulation and the charge of the bipolar stimulation pulses. The latter is defined by the product of current amplitude and pulse width. For this application, the stimulation frequency is fixed to $20 \mathrm{~Hz}$. The stimulator is capable of generating pulses with a current amplitude of up to $127 \mathrm{~mA}$ and a pulse width of up to $500 \mu \mathrm{s}$ (width of one phase of the bipolar pulse). However, for FES cycling with stroke patients only a current in the range of $I_{\min }=0$ to $I_{\max }=50 \mathrm{~mA}$ and a pulse width in the range of $p w_{\min }=100$ to $p w_{\max }=500 \mu \mathrm{s}$ is applied due to residual sensation of the patients. Then the range for the charge results in $0-25 \mu \mathrm{J}$. Mapping this to the range $0-1$ yields the normalized charge which can be adjusted for each stimulated muscle during a cycling exercise. Pulse width and current can be determined from a given normalized charge $Q_{n}$ by the following equations:

$$
\begin{aligned}
& p w=p w_{\text {min }}+\left(p w_{\text {max }}-p w_{\min }\right) \times \operatorname{sqrt}\left(Q_{n}\right) \\
& I=I_{\text {min }}+\left(I_{\text {max }}-I_{\text {min }}\right) \times \operatorname{sqrt}\left(Q_{n}\right)
\end{aligned}
$$

It should be noted that individual charges and thus pulse widths and current amplitudes are applied to each muscle. Charge control in this form liberates the therapist from choosing the pulse width and current separately, as in the classical approach. However, to date, it is unclear to the authors if the proposed sharing of pulse width and current amplitude for a given charge is optimal in terms of stimulation effect, sensation, and muscular fatigue. A deeper understanding of the underlying physiological principles of the electrical stimulation of nerves $[7,27]$ is required at this point. The charge control strategy was implemented on the PC which sends calculated pulse widths and current amplitudes periodically to the stimulator via the USB interface.

\section{Results}

The new amplifier was verified in cycling experiments with a healthy male subject. The study was approved by the Local Research Ethics Committee at Charité Berlin. Tests were performed under isokinetic conditions at a motor controlled cadence of $30 \mathrm{rpm}$ and each test contained around 15 crank revolutions. For each test, EMG profiles over the crank angle were computed together with confidence intervals $(68 \%)$. The EMG of each muscle was normalized between 0 (base line) and 1 (maximum EMG value during volitional cycling). Passive forces were initially measured while the subject was neither volitional cycling nor being stimulated but just being moved by the motor. For all following tests, the calculated active tangential forces at the crank arms were displayed in real time to the cyclist who was instructed not to exceed $40 \mathrm{~N}$ when cycling volitionally. This corresponds to a maximum crank torque of $8.8 \mathrm{Nm}$. In the first trial (Test A), the subject was asked to cycle volitionally. During this test the dynamic stimulation ranges and measured EMG activation profiles were plotted in real time over the crank angle. The experimenter could adjust the start and stop angles of the stimulation pattern in steps of $5^{\circ}$ in order to make the stimulation pattern visually fit to the volitional EMG activation ranges while avoiding overlapping muscle activations. The obtained stimulation pattern at a cadence of $30 \mathrm{rpm}$ is given in Table 1. Following this step, low-intensity stimulation was applied using the selected stimulation pattern while the subject was passive (Test B). Stimulation intensities of all muscles were set to $50 \%$ of the maximal stimulation intensity (normalized charge: 0.5 , pulse width: $382 \mu \mathrm{s}$, current amplitude: $35 \mathrm{~mA}$ ). The stimulation frequency was $20 \mathrm{~Hz}$ in all stimulation tests. Self-adhesive electrodes of size $7.5 \times 13 \mathrm{~cm}$ were used on the quadriceps and hamstring on both legs to stimulate the muscles and to detect EMG activity. After Test 

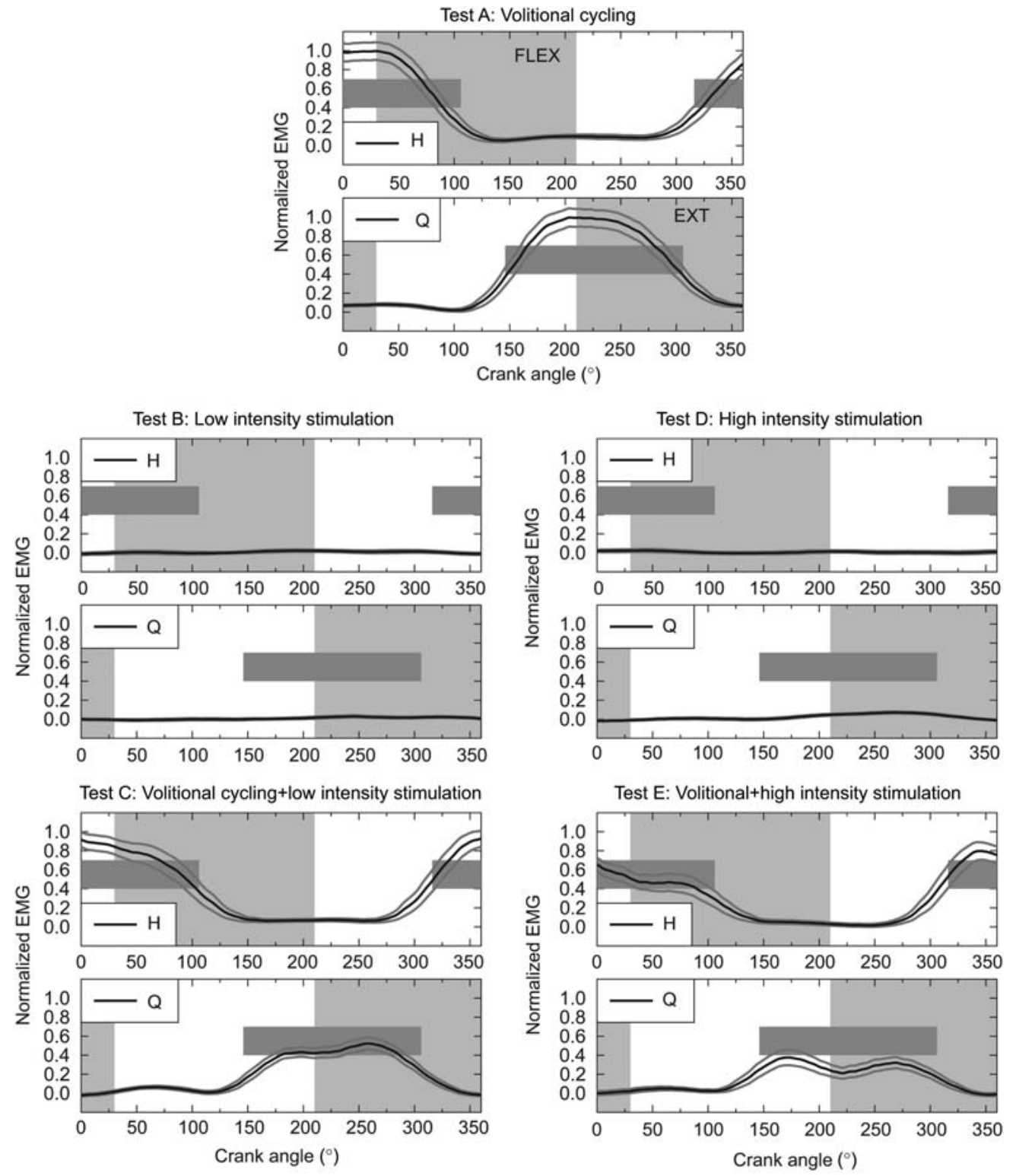

Figure 4 EMG profiles of the hamstring $(\mathrm{H})$ and quadriceps $(\mathrm{Q})$ of the left leg for all cycling tests (A-E). The ranges of knee flexion and extension are shown in light gray. The used stimulation pattern at a cadence of $30 \mathrm{rpm}$ is drawn in dark gray.

$\mathrm{B}$, the subject was asked to cycle voluntarily while the lowlevel stimulation was still active (Test C). Subsequently, the stimulation intensity was increased to the maximum level (normalized charge: 1.0, pulse width: $500 \mu \mathrm{s}$, current amplitude: $50 \mathrm{~mA}$ ) for all muscles while the subject was not actively cycling (Test D). Finally, while keeping maximum stimulation intensity, the subject was instructed to cycle additionally by himself (Test E).

In the following section, only data from the left leg are reported. In Figure 4, the normalized EMG profiles are presented for Tests A-E. The EMG-aligned dynamic stimulation ranges, chosen by the experimenter in Test A, are additionally shown in Figure 4. In Figure 5, recorded tangential forces are reported. Table 2 provides the average EMG of the hamstring and quadriceps for all tests and mean tangential forces during the extension and flexion phase of the knee, all compared to the values obtained during Test A.

\section{Discussion and conclusions}

The presented illustrative results show that a multichannel EMG measurement from stimulation electrodes is feasible during FES cycling. Also, under active superimposed electrical stimulation, muscle activity can be clearly detected. The EMG profiles obtained during volitional cycling (both with and without stimulation) are typical for cycling, showing a reciprocal activity fitting to extension and flexion of the knee joint. The phase advance of the EMG activities compared to the extension and flexion of the knee joint is 


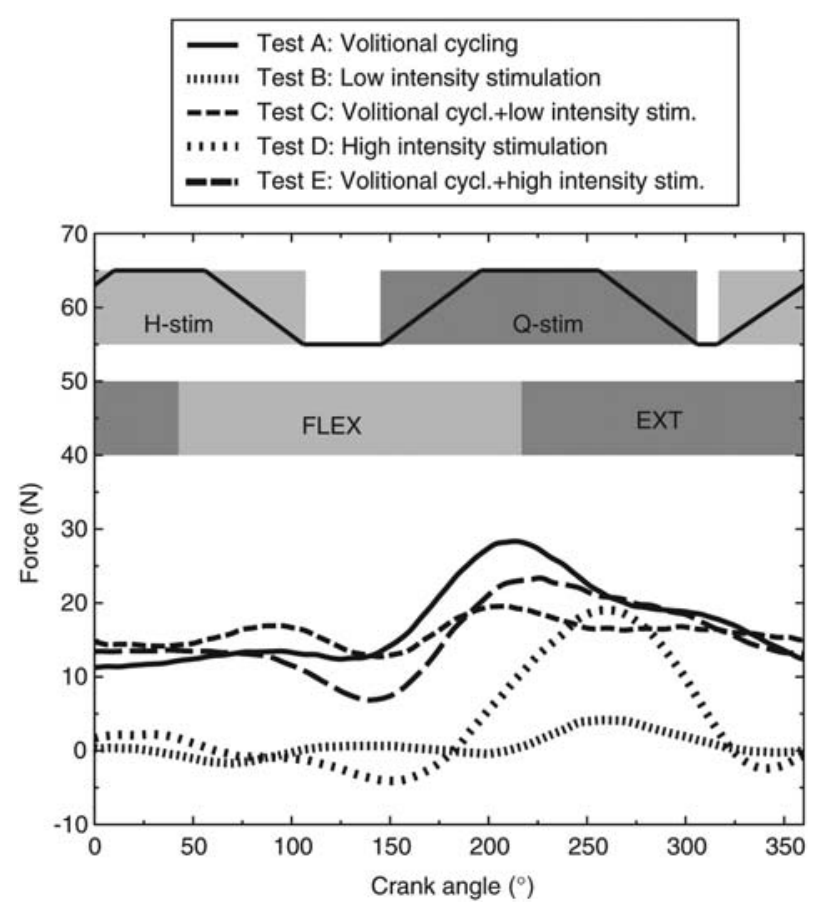

Figure 5 Tangential forces recorded at the left crank arm for all cycling tests (A-E).

due to time delay between muscle activation and force generation. The observed small overlapping of the EMG activities of hamstring and quadriceps can furthermore be explained by the fact that $M$. rectus femoris also flexes the hip and both stimulated hamstring muscles also extend the hip. In addition, a significant reduction in volitional EMG activity of the quadriceps can be observed under stimulation (more than 50\% decrease). Electrical stimulation of the quadriceps clearly contributes to the force generation, so that with stimulation the subject needs less effort to achieve force levels comparable to Test A (purely volitional cycling). Not much force generation was observed during hamstring stimulation (see results of Tests B and D), so that no strong change in EMG activity related to electrical stimulation was present.

The effectiveness of suppressing stimulation artifacts can be assessed from Tests B and C. Ideally, no EMG activity should be recorded during these tests. The observed average EMG values (see Table 2) of 2\%-5\% are acceptable and might be caused by remaining minor stimulation artifacts or unintended muscular activities of the subject. Relaxing perfectly under stimulation is practically impossible. The aim of this study was to prove the feasibility to measure volitional/reflex related EMG during FES cycling. Recruiting a healthy subject was the preferred option for this aim as such a subject can relax better during stimulation than a stroke patient and should not show disturbed reflexes. For these reasons, one can assume that recorded EMG activities during the stimulation tests without volitional cycling (Tests B and D) are mostly linked to stimulation artifacts. Following the instructions given during tests might also be a problem for some stroke patients. However, more crucial is the fact that the developed amplifier will later be used in therapy only with stroke patients in whom EMG activities of weak muscles are lower than in healthy subjects. To address this fact, the healthy subject was asked to generate low volitional tangential forces $(<40 \mathrm{~N})$ during the tests comparable to the values observed in stroke patients [18].

Disadvantages of the presented EMG amplifier are the limited frequency band of $170-700 \mathrm{~Hz}$ and extensive blanking periods ( $30 \mathrm{~ms}$ of every stimulation period) in order to suppress stimulation and switching artifacts. However, studies published by another research group have shown that a detection of volitional activity in stroke patients can be done by using an even more narrow frequency band of 330$460 \mathrm{~Hz}$ [11, 13, 26]. Unfortunately, M-waves cannot be assessed by using the EMG amplifier presented here due to the long muting periods. This represents a major drawback when measuring EMG from stimulation electrodes.

In a next step the EMG amplifier will be extensively used during FES cycling with stroke patients to monitor volitional activities and reflexes while performing the exercise. First successful tests have already been performed. Based on the results, biofeedback training will be developed for treatment of muscle imbalance in patients with spastic hemiparesis similar to the approach described in [3] but extended by FES. One currently unsolved problem is to distinguish crosstalk and co-contraction for patients with thin legs when using the presented amplifier.

When interpreting the EMG recordings, it must be taken into account that these EMG signals are measured through large electrodes covering muscle groups rather than individual muscles. Only a net EMG from several muscles is available which does not allow to draw precise conclusions on the activity of individual muscles. By contrast, such EMG recording from the healthy leg might be more suitable to tune the stimulation ranges in FES cycling than EMG patterns

Table 2 Average EMG values for the left quadriceps $\left(\mathrm{EMG}_{\mathrm{LQ}}\right)$ and left hamstring $\left(\mathrm{EMG}_{\mathrm{LH}}\right)$ as well as average tangential forces at the left crank during knee extension $\left(\mathrm{F}_{\mathrm{EXT}}\right)$ and flexion phase $\left(\mathrm{F}_{\mathrm{FLEX}}\right)$.

\begin{tabular}{lcccc}
\hline Test & EMG $_{\mathrm{LQ}}(\%)$ & $\mathrm{EMG}_{\mathrm{LH}}(\%)$ & $\mathrm{F}_{\mathrm{EXT}}(\%)$ & $\mathrm{F}_{\mathrm{FLEX}}(\%)$ \\
\hline Test A: volitional cycling & 100 & 100 & 100 & 100 \\
Test B: low-level stimulation & 2 & 2 & 8 & -2 \\
Test C: volitional cycling + low-level stimulation & 48 & 106 & 2 & 97 \\
Test D: high-level stimulation & 5 & 80 & 44 & -3 \\
Test E: volitional cycling +high-level stimulation & 37 & 95 & 80 \\
\hline
\end{tabular}

All variables are normalized with regard to the average values of Test A (volitional cycling without stimulation). 
taken from individual muscles using standard electromyographic kinesiology. Our preliminary results indicate that such an approach might work at least for the quadriceps. A positive drive torque at the crank was measured for the EMG-tailored stimulation range. However, using the stimulation range determined from hamstring EMG activity did not produce much force despite the fact that the EMG recorded from the same electrodes shows clearly visible EMG profiles.

Using smaller electrodes will improve the EMG measurement from stimulation electrodes as less charge will be accumulated after stimulation and therefore a reduction of muting periods will be possible. However, decreasing the electrode size will increase stimulation current densities underneath the electrodes, which can make the stimulation more unpleasant for the patient.

On the technological side, the stimulation device and the EMG amplifier have to be integrated into one physical device.

In addition to the EMG amplifier, force sensors have been included in the presented FES cycling system to assess the force produced by electrical stimulation. This allows automatic symmetry control in FES cycling. In contrast to normal FES cycling, the outlined feedback control concept excludes patients with a marked spasticity and/or increased muscle tone as well as patients unable to relax their leg muscles.

Another feature of the used FES cycling system is the use of charge control for a simple setting of the stimulation intensity. The feasibility of the proposed charge control approach has to be proven in future studies with patients.

\section{Acknowledgements}

This research was partly funded by the German Federal Ministry of Education and Research (BMBF) within the project RehaRobES (Grant 01EZ0766) and by an Egyptian Government scholarship held by Raafat Shalaby. The authors thank Prof. Stefan Hesse and Cordula Werner from the Medical Park Humboldtmühle Berlin, Department of Neurological Rehabilitation at the Charité-University Medicine Berlin, Germany for their advice during the development of the EMG amplifier and further cooperation.

\section{References}

[1] Ambrosini E, Ferrante S, Schauer T, Ferrigno G, Molteni F, Pedrocchi A. Design of a symmetry controller for cycling induced by electrical stimulation: preliminary results on postacute stroke patients. Artif Organs 2010; 34: 663-667.

[2] Bolton D. Electromyogram-triggered neuromuscular stimulation and stroke motor recovery of arm/hand functions: a meta-analysis. J Neurol Sci 2004; 223: 121-127.

[3] Brown DA, DeBacher GA. Bicycle ergometer and electromyographic feedback for treatment of muscle imbalance in patients with spastic hemiparesis: suggestion from the field. Phys Ther 1987; 67: 1715-1719.

[4] Brown DA, Nagpal S, Chi S. Limb-loaded cycling program for locomotor intervention following stroke. Phys Ther 2005; 85: $159-168$
[5] Chen C, He Z, Hsueh Y. An EMG feedback control functional electrical stimulation cycling system. J Signal Process Syst 2009; DOI: 10.1007/s11265-009-0425-5.

[6] De Luca CF, Knaflitz M. Surface electromyography, what's new? Turino, Italy: CLUT Publishers 1992.

[7] Durand DM. Electric stimulation of excitable tissue. In: Bronzino JD, editor. The biomedical engineering handbook. 2nd ed. Boca Raton, FL: CRC Press LLC 2000, pp. 1-22.

[8] Eigler F. Functional electrical stimulated leg cycling (FESLCE) in rehabilitation of patients after stroke. Dissertation, Ruprecht-Karls-Universität Heidelberg, Heidelberg, Germany 2009 (in German).

[9] Ferrante S, Pedrocchi A, Ferrigno G, Molteni F. Cycling induced by functional electrical stimulation improves the muscular strength and the motor control of individuals with post-acute stroke. Europa Medicophysica-SIMFER 2007 Award Winner. Eur J Phys Rehabil Med 2008; 44: 159-167.

[10] Frigo C, Ferrarin M, Frasson W, Pavan E, Thorsen R. EMG signals detection and processing for on-line control of functional electrical stimulation. J Electromyogr Kinesiol 2000; 10: $351-360$

[11] Fujiwara T, Kasashima Y, Honaga K, et al. Motor improvement and corticospinal modulation induced by hybrid assistive neuromuscular dynamic stimulation (HANDS) therapy in patients with chronic stroke. Neurorehabil Neural Repair 2009; 23: 125-132.

[12] Hankey GJ, Warlow CP. Treatment and secondary prevention of stroke: evidence, costs, and effects on individuals and populations. Lancet 1999; 354: 1457-1463.

[13] Hara Y, Ogawa S, Muraoka Y. Hybrid power-assisted functional electrical stimulation to improve hemiparetic upperextremity function. Am J Phys Med Rehab 2006; 85: 977985.

[14] Janssen TW, Beltman JM, Elich P, et al. Effects of electric stimulation-assisted cycling training in people with chronic stroke. Arch Phys Med Rehabil 2008; 89: 463-469.

[15] Kamono A, Kotaka Y. Development of gait assist device for foot drop patients. In: Triolo RJ. editor. Proceedings of the 6th Annual Conference of the International Functional Electrical Stimulation Society, Cleveland, Ohio, USA 2001: 178180.

[16] Kamono A, Muraoka Y, Tomita Y, Shimaoka H, Uchida S, Ota T. Development of EMG-controlled electrical stimulation system. In: Proceedings of the 41st SICE Annual Conference. Osaka, Japan: IEEE 2002; 3: 2022-2027.

[17] Katz-Leurer M, Sender I, Keren O, Dvir Z. The influence of early cycling training on balance in stroke patients at the subacute stage. Results of a preliminary trial. Clin Rehabil 2006; 20: 398-405.

[18] Kautz SA, Duncan PW, Perera S, Neptune RR, Studenski SA. Coordination of hemiparetic locomotion after stroke rehabilitation. Neurorehabil Neural Repair 2005; 19: 250-258.

[19] Knaflitz M, Merletti R. Suppression of simulation artifacts from myoelectric-evoked potential recordings. IEEE Trans Biomed Eng 1988; 35: 758-763.

[20] Langzam E, Isakov E, Mizrahi J. Evaluation of methods for extraction of the volitional EMG in dynamic hybrid muscle activation. J Neuroeng Rehabil 2006; 3: 27.

[21] Lee M, Kilbreath SL, Singh MF, et al. Comparison of effect of aerobic cycle training and progressive resistance training on walking ability after stroke: a randomized sham exercisecontrolled study. J Am Geriatr Soc 2008; 56: 976-985.

[22] Meilink A, Hemmen B, Seelen H, Kwakkel G. Impact of EMG-triggered neuromuscular stimulation of the wrist and 
finger extensors of the paretic hand after stroke: a systematic review of the literature. Clin Rehabil 2008; 22: 291-305.

[23] Merletti R, Knaflitz M, De Luca CJ. Electrically evoked myoelectric signals. Crit Rev Biomed Eng 1992; 19: 293-340.

[24] Metting van Rijn AC, Peper A, Grimbergen CA. High-quality recording of bioelectric events. Med Biol Eng Comput 1990; 28: 389-397.

[25] Minzly J, Mizrahi J, Hakim N, Liberson A. Stimulus artefact suppressor for EMG recording during FES by a constant-current stimulator. Med Biol Eng Comput 1993; 31: 72-75.

[26] Muraoka Y. Development of an EMG recording device from stimulation electrodes for functional electrical stimulation. Front Med Biol Eng 2002; 11: 323-333.

[27] Rattay F. Electrical nerve stimulation: theory, experiments and applications. Vienna: Springer-Verlag 1990.

[28] Schauer T, Nahrstaedt H, Ambrosini E, Ferrante S. Ergometer system with electrical stimulation for training of lower limb motor coordination in stroke patients. In: Proceedings of the 2nd European Conference Technically Assisted Rehabilitation (TAR 2009). Berlin, Germany 2009. ISSN 18624871.

[29] Schauer T, Salbert R, Negaard N, Raisch J. Detection and filtering of EMG for assessing voluntary muscle activity during FES. In: Swain I, Taylor P, editors. Proceedings of the 9th Annual Conference of the International Functional Electrical Stimulation Society (IFESS 2004), Bournemouth, UK 2004: 185-187.

[30] Seki K, Sato M, Handa Y. Increase of muscle activities in hemiplegic lower extremity during driving a cycling wheelchair. Tohoku J Exp Med 2009; 219: 129-138.

[31] Sennels S, Biering F, Andersen O, Hansen S. Functional neuromuscular stimulation controlled by surface electromyographic signals produced by volitional activation of the same muscle: adaptive removal of the muscle response from the recorded EMG-signal. IEEE Trans Rehabil Eng 1997; 5: 195-206.

[32] Shalaby R, Nahrstaedt H, Schauer T, Liedecke W, Raisch J. Voluntary muscle activity detection using a single pair of electrodes for EMG-controlled FES. In: Proceedings of the 14th Annual Conference of the International Functional Electrical Stimulation Society (IFESS), Seoul, South Korea 2009: 69-71.

[33] Sheffler LR, Chae J. Neuromuscular electrical stimulation in neurorehabilitation. Muscle Nerve 2007; 35: 562-590.

[34] Stapelfeldt B, Mornieux G, Oberheim R, Belli A, Gollhofer A. Development and evaluation of a new bicycle instrument for measurements of pedal forces and power output in cycling. Int J Sports Med 2007; 28: 326-332.

[35] Szecsi J, Krewer C, Müller F, Straube A. Functional electrical stimulation assisted cycling of patients with subacute stroke: kinetic and kinematic analysis. Clin Biomech 2008; 23: 1086-1094.

[36] Thorsen R. An artefact suppressing fast-recovery myoelectric amplifier. IEEE Trans Biomed Eng 1999; 46: 764-766.

[37] Thorsen R, Ferrarin M, Spadone R, Frigo C. Functional control of the hand in tetraplegics based on residual synergistic EMG activity. Artif Organs 1999; 23: 470-473.

[38] Thorsen R, Ferrarin M, Veltink P. Enhancement of isometric ankle dorsiflexion by automyoelectrically controlled functional electrical stimulation on subjects with upper motor neuron lesions. Neuromodulation 2002; 5: 256-263.

[39] Thorsen R, Spadone R, Ferrarin M. A pilot study of myoelectrically controlled FES of upper extremity. IEEE Trans Neural Syst Rehabil Eng 2001; 9: 161-168.

[40] Thorsen RA, Occhi E, Boccardi S, Ferrarin M. Functional electrical stimulation reinforced tenodesis effect controlled by myoelectric activity from wrist extensors. J Rehabil Res Dev 2006; 43: 247-256.

[41] Webster J, editor. Medical instrumentation: application and design. 4th ed. New York: John Wiley \& Sons 2009.

[42] Yeom H, Park Y, Yoon H. Gram-Schmidt M-wave canceller for the EMG controlled FES. IEICE Trans Inf Syst 2005; E88-D: 2213-2217.

Received March 4, 2010; accepted November 3, 2010; online first December 17, 2010 